\title{
Une éducation salutogénique basée sur les capabilités des patients: une nouvelle composante de l'éducation thérapeutique
}

\author{
Olivia Gross* et Rémi Gagnayre
}

Laboratoire Éducations et Pratiques de Santé (LEPS, EA3412), Université Paris13, UFR SMBH, 74, rue Marcel-Cachin, 93017 Bobigny cedex, France

Reçu le 19 septembre 2017 / Accepté le 19 février 2018

\begin{abstract}
Résumé - L'éducation thérapeutique du patient vise à donner aux patients les connaissances et compétences d'auto-soins et d'adaptation à la maladie qu'ils estiment nécessaires pour mieux vivre avec leur maladie chronique. Indépendamment de l'ETP, des patients engagés comme acteurs sociaux dans le système de santé sont parvenus à un état salutogénique caractérisé par un sentiment de cohérence. De cette hypothèse découle celle de soutenir la salutogénèse des patients au moyen d'une éducation capabilitante développant leur capacité à influer sur le système de soins et en particulier sur leur environnement proche, en particulier sur les services de soins. Il s'agit d'ouvrir une nouvelle voie à l'ETP en y intégrant des séances d'éducation salutogénique qui visent à agir sur les vulnérabilités iatrogènes définies comme les situations provoquant des atteintes aux sécurités (physiques et psychosociales) des patients. Les patients apprendraient à utiliser leurs expériences de soins et celles de leurs pairs pour identifier les stratégies de changement contribuant à leur meilleure sécurité et ce faisant, aux missions des hôpitaux d'être des promoteurs de santé. De nouvelles structures pourraient voir le jour, les UTEEP: Unité Transversale d'Éducation et de l'Engagement du Patient.
\end{abstract}

Mots clés : ETP / capabilités / salutogénèse / sécurité des soins / engagement des patients

Abstract - A salutogenic education based on capabilities: a new part of patient education. The aim of therapeutic patient education (TPE) is to give patients the knowledge and competencies they feel they need, in terms of self-care and life skills, to cope with their chronic disease. Independent of TPE, patients engaged as social actors in the health system have achieved a salutogenic state characterized by a sense of coherence. Based on that assumption, patients' salutogenesis should be supported via education that enables them to develop the ability to influence both the health system and their immediate environment-especially healthcare services. A new avenue could be created for TPE by incorporating salutogenic education sessions aimed at impacting iatrogenic vulnerabilities, defined as situations that compromise patients' (physical and psychosocial) safety. Patients would learn to use their own healthcare experiences and those of their peers to identify change strategies contributing to their greater safety and, in so doing, to the hospitals' mission as health promoters. New entities could be created: UTEEPs, or Unités Transversales d'Éducation et de l'Engagement du Patient (Crosscutting Patient Education and Engagement Units).

Key words: patient education / capabilities / salutogenesis / healthcare safety / patient engagement

\section{Problématique}

L'éducation thérapeutique du patient vise à donner aux patients les connaissances et compétences d'auto-soins et d'adaptation à la maladie qu'ils estiment nécessaires pour mieux vivre avec leur maladie chronique [1]. Depuis la loi HPST de 2009 [2], elle est inscrite dans le parcours de soins et

\footnotetext{
$\overline{\text { *Auteur de correspondance }}$ : olivia.gross@univ-paris13.fr
}

est proposée aux patients majoritairement à l'hôpital lors de séances dédiées [3]. Malgré ses références aux théories éducatives, elle souffre de remises en question qui pointent son formalisme [4], sa pédagogie par compétences [5], la standardisation trop normée des séances éducatives et dans certains cas, leur prisme trop biomédical [3].

Pourtant, bien menée, elle est centrée sur les attentes des patients et les autonomise [6] en les outillant dans une perspective autonormative [7]. Et, ce qui n'est pas le moindre de ses mérites, elle a largement contribué à ce que les soignants 
partagent leurs savoirs avec les patients, à ce que les connaissances soient vues comme thérapeutiques, dans un contexte où elles étaient jusqu'alors considérées comme anxiogènes. Enfin, elle permet une meilleure implication des patients dans leurs soins, ce qui participe de leur mieux-être [6]. Indépendamment de l'ETP, des patients ont, par euxmêmes, acquis un certain nombre de savoir-faire qui, malgré le fait qu'ils aient expérimenté une vulnérabilité initiale importante, ont contribué à les réconcilier avec leur vécu de malades chroniques. Il s'agit de patients, dits patients-experts $[8,9]$, patients-ressources [10] ou bien encore de patientspartenaires [11] qui se sont engagés dans le système de santé afin de contribuer à l'améliorer et qui ont réussi à transformer les pratiques dans les champs dans lesquels ils se sont engagés. Ils se sont réconciliés avec leur histoire de vie, et on peut avancer l'idée que c'est en grande partie grâce à leur rôle d'acteur social.

Il s'agit dans cet article de comprendre le processus qui a commué leur vulnérabilité initiale en un état que nous définirons plus bas de salutogénique, et de voir en quoi ce même processus pourrait fertiliser l'éducation thérapeutique.

L'hypothèse centrale qui guide notre proposition est celle selon laquelle le souci des autres (des pairs et/ou du système de santé), dès lors que les patients sont engagés au niveau collectif, sert secondairement le souci de soi. Ce projet étant conforté par le fait que les malades éprouvent naturellement un désir de mettre leur expérience au service des autres [12].

De cette hypothèse découle celle de développer chez les patients au moyen d'une éducation salutogénique le souci des autres, ce qui passe par leur donner, à partir d'une conscientisation de l'in-juste (à savoir des situations de soins qui leur paraissent inadaptées), la capacité à influer sur le système de soins et en particulier sur les services de soins qu'ils côtoient.

Cet article reprend en première partie les principales leçons apprises auprès des patients qui s'engagent comme acteurs de santé dans le cadre mouvant de la démocratie sanitaire en les interrogeant sous l'angle des capabilités individuelles et collectives. Dans une deuxième partie, il dessine les rapprochements entre une éducation salutogénique et l'éducation thérapeutique. Enfin dans une troisième partie, il fournit des pistes d'une forme d'opérationnalisation à un moment où le choix programmatique de l'ETP nécessite de nouvelles impulsions.

\section{L'engagement salutogénique des patients}

\subsection{De la vulnérabilité à la salutogénèse}

Il faut voir l'engagement des patients et de leurs proches dans le système de santé comme un levier d'amélioration de la qualité des soins [13] en ce sens que leur engagement est vecteur de transformations. Cela se comprend au regard des raisons qui les ont poussés à s'engager. La maladie chronique confronte les malades et leurs proches à leurs limites. Quand la personne est malade chronique de naissance, elle doit sans cesse les revisiter, sous l'effet cumulé de ses aspirations et de la maladie qui toutes deux évoluent. S'ajoutent à ces vulnérabilités, tant physiques que psychologiques, des vulnérabilités iatrogènes, induites par le système de soins [14] malgré les meilleures intentions des soignants. Les patients (et cela inclut leurs proches) qui s'engagent comme acteurs du système de santé ont tous ressentis ces différentes vulnérabilités et ils ont de surcroît éprouvé un sentiment d'inefficacité d'autrui (que ce soit celle d'un individu, d'un système ou de la science) dans le champ qui a précipité leur engagement [9]. D’engagements en engagements, ils ont conçu et mis en œuvre des actions correctrices et y ont gagné en sentiment de compétences. Ils mettent leurs apports au profit de leurs pairs, c'est-à-dire au profit des personnes dans lesquelles ils se reconnaissent. Ce groupe, plus ou moins large, se constitue à partir d'un sentiment d'affiliation et selon des critères qui sont de l'ordre de l'impensé. Ainsi, par exemple, dans le même contexte, les uns s'identifient à l'hémophilie, tandis que les autres s'identifient aux maladies rares, ou au VIH. À notre connaissance, il n'y a pas d'étude qui ait cherché à comprendre ces différences d'attractivité identitaire.

Les patients engagés (PE), dont certains méritent amplement d'être qualifiés de patients-experts, agissent poussés par une énergie qui les dépasse et qui gagne en force et en qualité en même temps qu'elle se commue en passion pour améliorer les connaissances (passion cognitive) [15] et/ou pour bousculer l'établi (passion conative, en ce sens qu'elle est un moteur pour l'action [8]). Le caractère passionnel de ces pulsions cognitives et conatives les conduit à vouloir tout connaître des domaines qui mobilisent leur engagement, et dans un deuxième temps à vouloir les transformer de manière à les conformer à leurs attentes :

- la passion cognitive les conduit à produire des savoirs qui sont protéiformes: il peut s'agir d'auto-clinique [16,17], voire d'auto-clinique par procuration quand ces savoirs portent sur un proche, de savoirs construits collectivement dans un contexte associatif [18], de savoirs élaborés grâce à la fréquentation des forums de discussions sur Internet [1921]. Les savoirs produits par les PE s'affranchissent le plus souvent des règles scientifiques (sauf quand des patients mènent des études scientifiques). Ce sont des savoirs émancipateurs, en ce sens qu'ils cherchent à compléter les savoirs savants [22]. Il s'agit de savoirs militants, libérés des dogmes médico-scientifiques, de savoirs qui revendiquent une preuve par les faits [23] autrement dit, il s'agit d'un savoir de l'épreuve versus un savoir des preuves;

- la passion conative conduit les PE à devenir des acteurs sociaux afin de mettre en œuvre des actions correctrices. Ils ont, comme les acteurs sociaux [24], un pouvoir réflexif qui dépend de leurs interactions sociales. Ils savent que si la vulnérabilité est individuelle, ses réponses sont collectives [25]. Pour mener des actions efficaces, ils doivent s'inscrire dans des relations sociales et des relations de pouvoir, relations qui sont définies comme étant: " toute chance de faire triompher au sein d'une relation sociale, sa propre volonté, même contre des résistances » [24]. Cette mutation, qui voit des personnes en situation de vulnérabilité devenir des acteurs sociaux s'opère sous la combinaison de trois principes : (1) la capacité de l'acteur collectif à se reconnaître comme acteur social apte à changer la société dans son ensemble; (2) la capacité à identifier ceux qui ont une autre vision sociale; (3) la capacité à concevoir l'enjeu sous ce rapport afin de former son action en conséquence [26]. Les patients qui s'engagent comme acteurs du système de santé sont force de proposition, ce qui ne va pas sans susciter des conflits. 
En effet, deux composantes principales déterminent le concept d'acteur social : le conflit et/ou la coopération [27]. Pour ce qui est des coopérations, les PE ont l'art de mettre en lien des professionnels isolés les uns des autres et ainsi de créer des opportunités de coopération entre eux. Ils ont aussi des personnes ressources dans leur entourage, qui sont soit des pairs qualifiés ou des professionnels, auprès de qui ils se tournent dans le but d'apprendre quelque chose ou de vérifier une information. En ce sens, ils sont experts de l'expertise des autres. Cette capacité à avoir accès à l'expertise des autres dépend en grande partie de la reconnaissance dont ils bénéficient: elle facilite les échanges propices aux apprentissages mutuels. Ce faisant, les patients engagés consacrent énormément de temps et d'énergie à leurs activités, ce qui est propice au développement de leurs savoir-faire.

Leur motivation d'effectance, les pousse à vouloir être efficaces et utiles : l'efficacité renvoyant à l'impact de leurs actions, et l'utilité à leur engagement au profit de leurs pairs [8]. Loin de les laisser envahis par la maladie, leur activité intense sert leur état psychologique: les "patients engagés » vont psychologiquement bien [9]. Leurs passions les animent et en tant que forces émotionnelles, elles ont compensé le gouffre émotionnel de la vulnérabilité. Dans un contexte défavorable, ils ont réagi, agi, et comme résultat de ce processus autopoiétique [28], ils se sont guéris sur le plan émotionnel. Si les épisodes de vulnérabilité ont été surmontés c'est qu'ils ont été mobilisés pour concevoir des actions correctrices qui répondent aux besoins constatés.

Cela laisse à voir un processus de salutogénèse [29] caractérisé par un sentiment de cohérence basé sur un sentiment de sens, un sentiment de compréhension et un sentiment de pouvoir gérer les évènements de la vie. Alors qu'ils étaient confrontés à une situation tout à fait anxiogène, leur engagement passionné a finalement donné du sens à ce qu'ils avaient traversé. Cela nécessite qu'ils aient problématisé leur vécu, à savoir qu'ils se soient posé une question salutogénique définie comme «la vraie question» par Antonovsky, question qui naît de la rébellion et oppose une résistance à l'acceptation aveugle [30].

Dans la même lignée, l'analyse de différents mouvements sociaux en santé (antipsychiatrie, disabilities studies) fait émerger d'après nos constats [31] au moins quatre notions communes : le refus de se soumettre à un pouvoir politique ou médical; 1'idée que le pouvoir sur les corps et les âmes par le système de soins génère une souffrance qui domine parfois celle générée par la maladie elle-même; le fait que les personnes concernées par des vulnérabilités en santé ont des savoirs d'expérience qui peuvent être utiles à leurs pairs; et le fait que leur activisme, que leur engagement au profit d'un groupe, contribue à leur salutogénèse, autrement dit au souci d'eux-mêmes.

La salutogénèse des «patients engagés » est étroitement liée à leur engagement comme acteurs sociaux et donc, à leur agencéité qui renvoie aux approches par les capabilités et plus précisément au fait de s'aider soi-même et d'influer sur le monde [32]. De plus, l'approche par les capabilités est salutogénique, en ce sens qu'elle est tournée vers les possibilités d'avoir une vie humaine accomplie [33] plutôt que vers les seuls besoins qui sont orientés vers les déficits à combler.

\subsection{Des capabilités individuelles aux capabilités collectives}

Dans l'objectif de soutenir dans le cadre de l'ETP les mêmes processus salutogéniques, il s'impose de clarifier des notions et de tenter au regard des définitions actuelles de discuter leur rapprochement. Cet exercice n'est pas simple, du fait de l'origine anglaise des termes utilisés et du fait que ceuxci aient déjà fait l'objet d'entreprises de traduction qui les ont stabilisés en français. Il en est ainsi du terme agencéité qui est imprégné de références culturelles au point qu'il n'existe pas de mot juste pour l'exprimer tel qu'il résonne pour un chercheur anglais [34].

Le concept de capabilité [35] connaît les mêmes difficultés de traduction. Il a émergé en réaction au bien humain réduit à son utilité ou à ses composantes matérialistes. Les capabilités individuelles servent de cadre d'analyse aux situations des personnes. Elles prennent en compte ce qu'une personne peut faire en fonction de ses préférences afin de parvenir à un mieux-être qualifié de «liberté de bien-être» [35] car elles correspondent à un accroissement des libertés d'être et de faire [36]. Elles sollicitent un triptyque de fonctionnements : avoir des préférences, être capable de les réaliser, et avoir la possibilité de le faire. Avoir des préférences est essentiel car il n'y a de liberté de bien-être que s'il y a des choix possibles [37] : en effet, avoir le choix de se déplacer et choisir de ne pas le faire est bien différent d'avoir à subir cette situation.

Mais les capabilités et les préférences ne convergent pas toujours. Ainsi, ce n'est pas parce qu'on a envie de vivre d'une certaine manière qu'on s'en donne les moyens [37]. Et contrairement à ce que l'on pourrait penser, vouloir préférer ne va pas de soi: cela nécessite selon les auteurs une autre capabilité dite d'aspiration qui dépend autant de facteurs individuels (comme avoir confiance en soi, avoir une image de soi valorisée) que de la reconnaissance d'autrui. En effet, si autrui juge que dans une situation donnée, les aspirations individuelles sont déplacées, l'individu l'intègrera et n'osera pas aspirer. La possibilité d'atteindre ses préférences réclame la mise en œuvre d'une agencéité comme définie plus haut. De plus, selon Sen, les possibilités fonctionnelles sont socialement dépendantes. Elles reposent « sur le pouvoir interne de faire ou de choisir certaines choses et sur la disponibilité des circonstances environnementales et sociales, nécessaires à l'exercice d'une telle puissance » [33]. C'est en ce sens que les capabilités ne sont pas les attributs d'une personne mais celles d'une personne en interaction avec une collectivité et un contexte [38]. Cela passe par des facteurs de conversion qui favorisent l'actualisation des capabilités dans des fonctionnements effectifs : avoir théoriquement accès à quelque chose (par exemple à des soins dentaires) et ne pouvoir en réalité y accéder (par manque de dispositifs financiers facilement accessibles par exemple) traduit très concrètement des incapabilités liées à l'environnement.

Sen a distingué le bien-être occasionné par la satisfaction de voir aboutir ses combats pour le bien-être d'autrui (qui repose sur les capabilités individuelles d'engagement et de sympathie des agents) du bien-être purement individuel. D'autres auteurs que Sen ont poursuivi sa pensée et montré que des capabilités dites collectives articulaient les bien-être d'autrui et les bien-être individuels [36,39,40]. En effet, pour passer de ce qui est à ce qui pourrait être, pour développer 
pleinement les libertés, il est souvent nécessaire de mettre en œuvre des capabilités collectives [41] qui permettent des actions qui ne pourraient être réalisées par des individus seuls $[39,41]$. C'est le lien social qui permet l'exercice plein et entier de l'agencéité et qui permet des actions collectives qui in fine profitent aux intérêts individuels [39,40]. Mais cela n'est possible que si les individus développent une identité de groupe à partir d'une autre capabilité dite d'affiliation [42]. En santé, cette dernière renvoie en partie à la biosocialité qui dépend du sentiment d'une communauté de destin sur le plan biologique et/ou technologique [43]. Ainsi, si dans les maladies rares des avancées programmatiques ont pu avoir lieu, permettant notamment la législation sur les médicaments orphelins [44], c'est grâce au fait qu'au-delà de leurs différences, des liens aient été faits entre elles, ce qui n'allait pas de soi au départ.

Le plein déploiement des capabilités nécessite d'ajouter au triptyque de fonctionnements énoncé ci-dessus une étape que les capabilités individuelles ne permettent pas. Il s'agit de la conscientisation pleine et entière qui ne peut passer que par le groupe [45]. La conscientisation permet de produire des savoirs situés [46] à partir des savoirs expérientiels des vulnérabilités mis en commun. Les savoirs situés ont pour objectif d'influer sur le monde [47], à partir des subjectivités de l'éprouvé. De plus, la conscientisation appelle à des formations et actions collectives pour y répondre [45] afin de développer un pouvoir de faire mais aussi un pouvoir de faire ensemble. Si le groupe permet des capacités d'action qui diffèrent des capabilités individuelles du même registre c'est qu'il produit une intelligence collective et possède des dynamiques de pression [48]. L'intelligence collective permet à partir des interactions entre acteurs, d'octroyer rétrospectivement du sens aux évènements [49]. Et le groupe permet des revendications que l'individu seul ne peut mener: «l'organisation collective apparaît bien souvent comme une étape essentielle pour faire entendre sa voix » [36]. De même, les capabilités d'aspiration se développent mieux en groupe [50]. Ne serait-ce que parce qu'elles se répandent par capillarité au sein du groupe, y compris parmi les plus vulnérables qui sont connus pour être ceux qui ont le plus de difficulté à aspirer [50]. De plus, l'action de groupe est plus pertinente que les actions isolées qui sont vite désordonnées: «il faut que ces expériences, ces révoltes isolées, se transforment en savoir commun et en pratiques coordonnées » [51]. Sans compter que dans un rapport de pouvoir à l'institution, un groupe est plus légitime que des individus isolés et l'agencéité collective mieux accueillie que les agencéités individuelles susceptibles d'être facilement ignorées ou marginalisées. Les rapports de pouvoir peuvent néanmoins être compensés par des institutions capabilitantes qui soutiennent les capabilités afin d'inviter les acteurs sociaux à les questionner. Cette démarche est d'ailleurs celle promue par les principes de la démocratie sanitaire qui ont conduit à l'institutionnalisation des représentants d'usagers (RU) afin d'améliorer la qualité et la sécurité des soins. C'est important car on sait la durée des actions - et donc leur impact - dépend de leur institutionnalisation [40]. Grâce au mandat qui leur est attribué pour questionner les pratiques de soins, le pouvoir de faire et d'être des RU a gagné en amplitude et ce faisant, ils contribuent à ce que l'institution s'améliore, selon le principe fondateur de la démocratie sanitaire [13].

\section{Une éducation salutogénique comme fertilisateur de l'éducation thérapeutique}

Les patients engagés dans le système de santé se sont mis en mouvement pour apporter des réponses aux vulnérabilités qu'ils ont constatées, que ce soit dans leur propre parcours ou dans celui de leurs pairs. Parmi les vulnérabilités, certaines renvoient à l'in-juste, à savoir à des situations qui ne répondent pas aux besoins alors que leur satisfaction aurait pu être possible, ce qui, de surcroît, ne permet pas de déployer les capabilités. Le milieu hospitalier est en effet à de nombreux aspects hautement pathogène [52]. Cela appelle des actions correctrices et nécessite de pouvoir faire émerger le conflit entre des pratiques établies et des pratiques possibles. Lorsque ce sont des patients qui participent à ce processus, ils y gagnent à la fois en liberté et en sentiment de cohérence car cela permet rétrospectivement de justifier le vécu de l'in-juste, de lui donner $\mathrm{du}$ sens, et cela indépendamment des bénéfices individuels. Preuve en est que les plaintes qui remontent au niveau des directions hospitalières réclament demandent bien souvent des actions correctrices dont les plaignants ne disposeront pas eux-mêmes. Il y est souvent fait état qu'ils agissent pour les autres, pour que le préjudice qu'eux-mêmes ont subi ne se reproduise pas.

Les vécus de l'in-juste doivent déboucher sur des expériences de l'in-juste [51] (cet auteur parle «du vécu et de l'expérience d'injustice» mais la notion d'injustice dans le contexte des soins renvoyant implicitement à l'absence d'équité, la terminologie «in-juste» parait plus appropriée à la démonstration qui suit). Il s'agit de conscientiser les différents enjeux sur les plans normatifs et relationnels et d'identifier les actions correctrices à porter. Or cela ne va pas de soi : l'in-juste n'apparaît comme tel qu'à ceux qui sont prêts à questionner les pratiques et à s'autoriser à aspirer. Or, « ceux qui sont privés de tout n'ont pas le courage de désirer beaucoup et, sur l'échelle de la satisfaction des désirs, leurs privations sont rabaissées et perdent toute valeur » [53]. La théorie de la reconnaissance $[54,55]$ est l'opérateur théorique principal de l'expérience de l'in-juste [51]. Il s'agit d'articuler auto légitimation [56] et reconnaissance institutionnelle. C'est là que l'institution hospitalière peut intervenir: il s'agit qu'elle reconnaisse les besoins, qu'elle accompagne les conscientisations, qu'elle les articule avec ses propres nécessités, qu'elle pense à panser ces souffrances, dans un souci d'amélioration continue de ses services. Le cadre de la démocratie sanitaire y participe. Mais cela reste au niveau des représentants d'usagers et certains y voient des limites [56,57]. Quant aux réponses aux questionnaires de satisfaction, elles ne sont pas suffisamment traduites en actions correctrices au niveau des services de soins. Et quand elles le sont, les répondants ne gagnent pas en salutogénèse car ils n'en sont pas informés, ces questionnaires étant anonymes et aucun retour sur les résultats et encore moins sur les éventuels changements n'étant organisé. Certes, les associations de malades se sont données comme fonction celle d'animer le recueil des expériences de soins et des vécus de l'ordre de l'in-juste, mais cela se passe en dehors du pouvoir instituant de l'institution dont le rôle est, comme cela a été vu, primordial dans l'actualisation des capabilités individuelles et collectives. Sans compter que les institutions ont un rôle tout autant fondamental que l'identité de groupe dans le soutien des capabilités collectives, car elles permettent de ne pas aspirer en 
vain et de réguler les rationalités individuelles et institutionnelles [58].

L'approche par les capabilités permet d'analyser les comportements des PE. Tous les PE se sont engagés de manière à passer de ce qui est à ce qui pourrait être. Ils ont influé sur leur environnement de manière à le conformer à leurs aspirations. Ils ont problématisé les phénomènes auxquels ils sont confrontés, ont aspiré à des voies innovantes, ils ont été capables de les mettre en œuvre et comme résultats de leur engagement, les pouvoir-faire ont gagné en possibilités. Et, ce faisant, ils ont eux-même gagné en salutogénèse. À partir de là, il s'agit de créer au profit d'un plus grand nombre de patients un environnement favorable au déploiement des capabilités du même registre. Or, le déploiement des capabilités requiert des actions de formation [36]. Parmi les pratiques qui pourraient y contribuer, il y a l'ETP. Rappelons que l'ETP vise à: «permettre aux patients d'acquérir et de conserver les capacités et compétences qui les aident à vivre de manière optimale avec leur maladie. Il s'agit par conséquent d'un processus permanent, intégré aux soins et centré sur le patient. L'éducation implique des activités organisées de sensibilisation, d'information, d'apprentissage, d'autogestion et de soutien psychologique, concernant la maladie, le traitement prescrit, les soins, le cadre hospitalier et de soins, les informations organisationnelles et les comportements de santé et de maladie» [1].

Si cette définition semble à la première lecture être centrée sur la gestion de la maladie au moyen d'un souci de soi, une deuxième lecture fait émerger l'importance du soutien psychologique. La notion de soutien, qui emprunte davantage à l'accompagnement qu'au registre de l'éducation, témoigne que l'ETP se veut être à la croisée de l'éducation et du Care. Mais le Care, malgré toutes ses qualités, n'est pas sans limites car, s'il est indispensable à la relation de soins, il ne laisse pas nécessairement de place à l'émancipation. Or, «il y a dans l'ETP, comme dans toute éducation une volonté d'émanciper les acteurs » [59]. Il y aurait donc possiblement un conflit entre l'éducation et le Care s'il n'était pris dans la définition qu'en donne Ricoeur, à savoir: "prendre soin avec et pour autrui dans des institutions justes» [60]. Définition qui impose de définir les institutions justes à partir des normes de ceux qui les expérimentent. L'ETP pourrait être le lieu pour éduquer aux capabilités collectives dans un souci d'amélioration des services de soins. Ainsi, l'ETP pourrait assurer ce qui est de sa spécificité, à savoir cette articulation care/éducation, et ce faisant assurer sa pérennité, tant: «son développement sera durable si l'ETP n'est pas utilisée pour conformer les patients à des normes» [59].

Ainsi, dans l'objectif de concilier le Care et l'éducation, d'articuler le «prendre soin» et «les institutions justes», et d'ouvrir une nouvelle voie à l'ETP, proposons-nous d'intégrer à l'ETP des séances d'éducation salutogéniques. Il s'agit d'associer les fonctionnements basés sur les compétences du souci de soi (qui ont un pouvoir thérapeutique) vers les capabilités collectives (qui ont un pouvoir salutogénique): l'action au profit d'un groupe décentrant de ses propres problèmes.

Si avec l'outil des compétences, l'ETP a été focalisée sur le souci de soi comme moyen de gestion de la maladie et du traitement, il faut lui associer un processus salutogénique qui fait appel aux ressources des individus.
Pour intégrer des séances salutogéniques à l'ETP, il est essentiel d'articuler capabilités et compétences, l'ETP, rappelons-le, s'étant construite sur le développement des compétences des patients. À notre connaissance, les liens entre les capabilités et les compétences ont été rarement faits, bien que certains travaux aient mentionné l'importance de compléter les approches par les compétences par celles par les capabilités [61]. Ce travail reste à être précisé pour déterminer précisément les zones de recouvrement entre les deux concepts dont les liens méritent d'être explorés, ne seraitce parce que compétences comme capabilités partagent l'objectif du mieux-être des personnes. L'enjeu est important dans la mesure où, si on s'accorde à réunir une éducation salutogénique et l'éducation thérapeutique, il faut éviter de perdre ce que les compétences ont permis de gagner c'est-àdire la clarté d'intention entre soignant-patient dans le rapport éducatif et le caractère opératoire de cette éducation.

Ainsi à ce stade, nous avançons quelques liens comme autant de présupposés, de possibles, articulations. Nous n'aborderons que les compétences individuelles [62] puisque ce sont les seules qui ont été formalisées dans le champ de l'ETP, à l'inverse des compétences collectives dont des travaux ultérieurs devraient montrer le lien avec les capabilités collectives.

Il est décrit dans le fonctionnement des capabilités, la possibilité d'atteindre ses préférences, afin de fonctionner de manière effective. Or, cette possibilité suppose des savoir-faire individuels qui pourraient correspondre aux compétences d'auto-soins et d'adaptation à la maladie $[6,63]$. Toujours dans les capabilités individuelles, nous avons mentionné que la possibilité d'atteindre une préférence réclame la mise en œuvre de facteurs de conversion et d'une agencéité qui fait référence à la possibilité d'action finalisée d'une personne. Or, il est admis qu'une compétence nécessite pour son acquisition et sa mobilisation d'activer des processus comme: la perception d'utilité de la compétence pour tendre vers un projet de réalisation, une confiance en soi, un sentiment de compétence, l'idée que l'on se fait de la représentation des autres sur sa compétence. Ces processus peuvent rapprochés de ce que les auteurs de la capabilité énoncent comme l'aspiration. Il faut pouvoir aspirer, autrement dit du point de vue de la compétence: se sentir en projet pour mettre en œuvre des compétences visées par l'ETP. Cela suppose que les processus décrits plus haut soient activés. Il y a donc un voisinage entre capabilité et compétences qui s'accentue dans la mesure où il a été montré qu'une compétence est toujours contextualisée [64]. Autrement dit, elle ne s'exprime que dans le contexte pour lequel elle est conçue et sa mobilisation dépend largement de conditions spécifiques. On peut citer l'importance de la reconnaissance par les autres acteurs de la compétence portée par un des leurs ainsi que les conditions matérielles favorisant sa réalisation. De plus, il est admis que pour mettre en œuvre une compétence, son auteur devrait savoir mobiliser son environnement, ses interactions sociales pour en favoriser l'application. Enfin, dans le domaine du transfert des acquis de formation, il est décrit le rôle majeur de l'entourage social qui doit accepter la modification de ses propres compétences pour permettre à la compétence de leur pair de prendre place dans l'organisation professionnelle ou sociale. Il y a donc dans la notion de compétence, une dimension contextuelle forte convoquant la nécessité d'un environnement favorisant 
l'expression compétence. C'est ce que l'on retrouve en partie dans les notions de facteurs de conversion ou d'environnement capabilitant. Enfin, on peut citer comme autre voisinage, l'importance des processus métacognitifs dans l'activation des compétences. Parmi ceux-ci, celui de prendre conscience de son expérience pour ajuster les nouveaux acquis et favoriser leur intégration. On peut envisager que cette prise de conscience agisse aussi bien au niveau de l'acquisition de la compétence que dans l'activation de la capabilité individuelle.

Mais pour autant, les deux notions, capabilités et compétences ne sont pas totalement substituables: ainsi, les approches par les capabilités ont, dès leur origine, valorisé la liberté des agents qui renvoie à ce qui pourrait être fait (notamment en fonction des facteurs de conversion), au regard de critiques sur ce qui est réellement possible ou sur ce qui est attendu. L'enjeu de la norme fait notamment partie de ce qui différencie la capabilité et la compétence. En effet, si la compétence n'est pas questionnée et traitée au regard des processus d'auto-normativité du patient, elle porte le risque d'une conformité sans recul par rapport aux attentes des patients.

\section{Le défi de l'opérationnalisation au sein d'un programme d'ETP}

Nonobstant les limites conceptuelles, il est possible de déterminer quelques repères pratiques montrant l'apport d'une éducation salutogénique (ES) à l'ETP. Avec des séances salutogéniques au sein de l'ETP, il s'agit de passer de la sollicitude à la sollicitation pour passer de ce qui est à ce qui pourrait être via une éducation aux capabilités collectives. Une ES diffère d'une éducation thérapeutique en ce sens que la dernière vise un meilleur bien-être des individus tandis que l'ES vise le bien commun. L'ETP développe les compétences individuelles concernant également l'entourage, l'ES développerait les capabilités collectives qui permettent d'agir de manière effective sur la conscientisation des expériences de l'in-juste et sur l'augmentation des libertés de choix. L'ETP vise des compétences psychosociales d'adaptation à la maladie; l'ES vise l'effectivité des libertés et les transformations de l'environnement et s'oppose à l'adaptation qui selon certains auteurs [65] ne se conclurait pas par un plein élargissement des opportunités. L'ETP augmente la confiance en soi, et l'estime de soi via le souci de soi ; l'ES les augmente via l'oubli de soi puisqu'il s'agit de servir l'intérêt général. Enfin, le succès de l'ETP s'évalue aux gains en termes de compétences, tandis que les objectifs de l'ES renvoient aux libertés de bien-être et à la salutogénèse.

\subsection{Les séances collectives d'une éducation salutogénique}

Sachant que l'ES a pour objectif concret l'amélioration des services de soins, ce type d'éducation doit être porté par une institution capabilitante car elle seule a le pouvoir nécessaire pour instituer les transformations de pratiques. Compte tenu que l'ES a été pensée autour des vulnérabilités iatrogènes, ses séances sont appelées dans un premier temps à se dérouler au sein des services hospitaliers et des cliniques, notamment celles consacrées aux soins de suite et de réadaptation (SSR).
De plus, l'ETP est basée dans certains programmes sur des séances éducatives collectives au sein de tels services qui sont justifiées pour des raisons pédagogiques (apprentissage collectif, conflits socio-cognitifs, apprentissage entre pairs). L'expérience du groupe, de sa dynamique sont pertinentes pour développer les capabilités collectives dans une perspective salutogénique.

Une éducation salutogénique participerait à l'émergence d'acteurs sociaux en les préparant à agir dans quatre domaines spécifiques.

\subsubsection{Créer une dynamique communautaire}

L'engagement dans un groupe dépend de la perception qu'un individu a de son identité [66]. Or, l'identité de groupe est absolument fondamentale, tant pour asseoir les revendications [41] que pour identifier les savoirs expérientiels généralisables ou les vulnérabilités sur lesquels il est possible d'agir. Il s'agirait donc de soutenir les capabilités d'affiliation des patients, notamment en leur apprenant à regarder ce qui les rassemble plutôt que ce qui les différencie et en organisant un processus démocratique de représentation. Pour les personnes que l'implication dans le collectif n'intéresse pas, le fait d'être exposé à une éducation salutogénique est une première expérience du collectif qui pourrait leur donner goût à la pair-émulation.

\subsubsection{Aspirer à une liberté d'agence}

La liberté d'agence ne peut se déployer que sous conditions. Il faut que les agents soient outillés afin d'être en mesure d'activer les capabilités d'aspiration. Un certain nombre d'auteurs rapportent l'importance de se référer à des théories de la justice [42] ou aux droits de l'homme [36]. En santé, les droits des malades, les codes de déontologie, les chartes et les bonnes pratiques éthiques, ou encore les normes concernant l'hygiène des soins sont autant de références nécessaires pour doter les patients d'une lecture critique de leurs expériences de soins.

\subsubsection{Apprendre à reconnaître les expériences de vulnérabilité iatrogènes}

Il s'agirait d'identifier les questions salutogéniques et de développer une praxis cognitive, à savoir une production collective de connaissances, à partir d'un sentiment d'identité communautaire [67]. Ces connaissances seraient basées sur les expériences de vulnérabilité iatrogènes, physiques comme psychosociales. Il faut intégrer l'idée que ces deux types de vulnérabilités renvoient à la sécurité des soins car, psychosociales comme physiques, elles portent atteinte à l'intégrité des personnes et peuvent conduire aux mêmes arrêts de soins. Pourtant, sauf de rares travaux [68], la sécurité des soins a tendance à n'être interrogée que sous l'angle des atteintes à la sécurité corporelle. Ce qui en fait un objet singulier au sein d'un système de santé qui se réfère au paradigme biopsychosocial de la santé. Il s'agit donc d'identifier les facteurs qui entravent la mission d'hospitalité de l'hôpital [69] dont fait partie la sécurité des soins. Cela nécessite de réaliser autour des expériences de l'in-juste une veille anticipative stratégique définie comme : « un processus collectif, proactif et continu, par lequel un groupe d'individus traque, de façon volontariste, et utilise des informations pertinentes concernant leur environnement exté- 
rieur et les changements pouvant s'y produire dans le but de créer des opportunités, d'innover, de réduire les risques et l'incertitude en général » [70]. L'ES serait l'occasion de former les patients à reconnaître les informations pertinentes, qui dans ce cadre concerneraient les épisodes de vulnérabilité iatrogènes, et à maîtriser les méthodes pour les repérer, les quantifier et les communiquer.

\subsubsection{Intégrer les questions salutogéniques et les réponses apportées dans les capabilités individuelles}

Ce type de séance devrait être consacré à repérer les facteurs de conversion capabilitants (comme mettre en place un numéro d'urgence qui facilite l'accès au service) et à identifier les nouvelles libertés qui en découlent.

Tableau 1. Exemples de fertilisation de compétences d'ETP par l'éducation capabilitante à visée salutogénique.

Table 1. Examples of fertilization of skills in therapeutic education via salutogenesis-enabling education.

Compétences en ETP (Gagnayre, d'Ivernois, Compétences/capabilités en ES Exemples de composantes
2010)

Exprimer ses besoins, ses sentiments relatifs à la maladie

Comprendre, s'expliquer

Solliciter l'aide de son entourage

Établir des liens entre la maladie et son histoire de vie

Repérer, analyser, mesurer (son état de santé)

Résoudre un problème de thérapeutique quotidienne, de gestion de sa vie et de sa maladie

Faire valoir ses choix de santé, formuler un projet

Utiliser le système de santé

Faire valoir ses droits

Utiliser les ressources de santé

Faire face, mettre en œuvre des conduites d'ajustement à la maladie, décider

Informer, éduquer son entourage/expliquer sa maladie
Conscientiser les vulnérabilités iatrogènes

Connaître les droits des malades, les principes éthiques, les chartes de bonne pratique, les codes de déontologie...

S'affilier à, développer son capital social

Établir des liens entre le sentiment de cohérence collectif et individuel

Repérer, analyser, mesurer (les vulnérabilités iatrogènes)

Résoudre les vulnérabilités

Aspirer, développer les options de santé au regard de l'organisation des soins, des prises en charges financières

Influer sur le système de santé

Penser la justice

Utiliser ses propres ressources et celles de ses pairs

Transformer ce qui peut l'être, identifier et mettre en œuvre ses préférences

Informer, éduquer son entourage
Identifier les évènements fragilisants à l'hôpital

Savoir qu'un deuxième avis médical, ne pas souffrir, consentir aux examens... sont des droits

Adhérer à une association de malades, échanger sur Internet

S'auto-légitimer à partir de ses accomplissements, avoir confiance en son propre jugement

Réaliser une veille anticipative stratégique, mener des enquêtes, des entretiens avec des personnes hospitalisées, analyser et diffuser les résultats

Identifier les questions salutogéniques comme la difficulté à accéder au service de soins dans des délais raisonnables

Concevoir des réponses alternatives comme l'accès direct au service de soins en cas d'urgence

Réaliser, des actions correctrices comme des affichettes sur l'hygiène

Identifier des reconnaissances de droits selon le point de vue des patients, les promouvoir auprès des soignants, institutions

Capitaliser sur ses points forts, apprendre de l'expérience d'un autre malade

Identifier les marges de liberté pour mettre en œuvre des changements, faire évoluer les prises en charge

Faire valoir sa démarche, sa contribution, auprès des autres malades 
Pour les patients qui auraient participé à tout le processus éducatif collectif, l'impulsion salutogénique collective devrait à ce stade irriguer les compétences/capabilités individuelles. La reconnaissance institutionnelle des vulnérabilités iatrogènes, physiques comme psychosociales ayant le pouvoir de faciliter la compréhension, le sentiment de pouvoir gérer les évènements de sa vie et de leur donner du sens. Les soignants éducateurs pourraient accompagner ce processus en mobilisant les fonctions métacognitives des patients dont on connait l'importance pour aider les apprenants à se mettre en projet. De plus, comme cela a été souligné [29], le lien entre le sentiment de cohérence collectif et le sentiment de cohérence individuel mérite d'être questionné, ce qui pourrait être fait lors de cette séance éducative.

\subsection{L'intégration des séances d'ES au sein des programmes d'ETP}

A priori, l'ES se déroulerait dans le cadre du suivi éducatif de groupe lorsque l'expérience de la maladie et du traitement, du rapport au système de soins est constituée. On peut envisager deux formats qui reposeraient sur l'animation par un binôme constitué par un professionnel et d'un patient intervenant. Ces deux formats ne sont pas exclusifs et peuvent être envisagés complémentairement en fonction des temps dont on dispose :

- un format intégré, qui articulerait les compétences habituellement visées par l'ETP aux capabilités décrites (Tab. 1). À condition de développer les marges de liberté, les compétences d'auto-soins comme les compétences d'adaptation [71] pourraient intégrer des objectifs pédagogiques renvoyant aux capabilités. Par exemple, sachant qu'une des compétences figurant dans le référentiel de l'ETP est de savoir utiliser les services de santé, cette compétence pourrait évoluer de manière à devenir : utiliser et influer sur les services de santé. Faire valoir ses droits peut aussi être entendu de manière individuelle dans un rapport soignant-patient comme d'une manière collective en contribuant à des reconnaissances de droit (comme celui d'une voie alternative au passage systématique par les urgences des personnes suivies pour un cancer qui ont besoin d'être réhospitalisées), ce qui pourrait s'exprimer comme : penser la justice;

- une voie dite juxtaposée, qui ajouterait des séances d'ES aux séances proposées aux patients dans le cadre des programmes hospitaliers.

En toute logique avec le principe du diagnostic éducatif, ce serait aux patients de choisir avec les soignants leur parcours d'ETP.

\section{Conclusion}

L'ETP a une force instituante sans pareil, ne serait-ce que grâce au fait qu'elle se déroule majoritairement au sein de l'institution hospitalière. Ses acteurs sont de fait des agents de changements et l'intégration de séances salutogéniques dans l'ETP pourrait s'inscrire dans ce prolongement. Il s'agit de promouvoir la salutogénèse conjointe des services de soins et de leurs utilisateurs au moyen d'une éducation salutogénique. Cette dernière contribuerait de par ses caractéristiques à combattre les vulnérabilités iatrogènes au moyen d'une éducation à une veille anticipative stratégique et aux règles de droit et de bonnes pratiques. Ce faisant, les acteurs professionnels de l'ETP apprendraient également des participants, et cette posture bénéficierait à l'ensemble de leurs activités d'ETP et de soins [72]. Ainsi, l'ETP participerait à la mission des hôpitaux promoteurs de santé et à la démocratie sanitaire. Une des concrétisations possibles de cette fertilisation d'une éducation salutogénique à l'ETP, outre les programmes d'ETP, pourrait se faire au niveau des Unités Transversales d'Éducation du Patient. À côté du soutien méthodologique qu'elles apportent aux soignants et plus récemment aux patients intervenants en ETP, ces structures pourraient être le lieu du soutien à l'éducation salutogénique, porte d'entrée selon nous à un soutien plus large pour l'engagement des patients. Par cette fertilisation, de nouvelles structures pourraient voir le jour: les Unités Transversales d'Éducation et de l'Engagement du Patient (UTEEP).

Cependant, comme toute innovation pédagogique, celles proposées dans cet article devront être accompagnées par un faisceau de recherches et être évaluées au niveau des libertés des acteurs sociaux, notamment en termes de transformations produites par les actions collectives, ainsi qu'au niveau des libertés de bien-être individuelles qui en découlent.

Conflits d'intérêts. Les auteurs déclarent n'avoir aucun lien d'intérêt concernant les données publiées dans cet article.

\section{Références}

1. World Health Organization, regional office for Europe. Therapeutic Patient Education: continuing education programmes for health care providers in the field of prevention of chronic diseases; 1998.

2. Loi n ${ }^{\circ} 2009-879$ du 21 juillet 2009 portant réforme de l'hôpital et relative aux patients, à la santé et aux territoires; 2009.

3. HCSP. Évaluation des programmes d'éducation thérapeutique des patients 2010-2014. Paris: Haut Conseil de la Santé Publique; 2015.

4. Inspection Générale des Affaires Sociales. Pertinence et efficacité des outils de politique publique visant à favoriser l'observance; 2015.

5. Grimaldi A. Où va l'Éducation Thérapeutique du Patient? Med Mal Metabol 2017; 11:606-607. DOI: 10.1016/S1957-2557(17) 30143-8.

6. d'Ivernois J-F, Gagnayre R. Apprendre à éduquer le patient : approche pédagogique : l'école de Bobigny. Paris : Éd. Maloine; 2016.

7. Barrier P. L'autonormativité du patient chronique: un concept novateur pour la relation de soin et l'éducation thérapeutique. ALTER 2008; 2:271-291. DOI: 10.1016/j.alter.2008.07.001.

8. Gross O, Gagnayre R. Le rôle de la passion dans la logique de l'agir des patients-experts. In: Nouvelles interventions réflexives dans la recherche en santé : savoirs expérientiels des malades et formation par la recherche en sciences infirmières, 2014 (E Jouet, O Las Vergnas, E Noelle-Hureaux, Eds), Paris, Éd. des Archives Contemporaines. 
9. Gross O, Gagnayre R. What expert patients report that they do in the French health care system, and the competencies and personality traits required. Educ Ther Patient - Ther Patient Educ 2014; 6:20104. DOI: 10.1051/tpe/2014014.

10. Chouleur F, Gendarme S, Ayav C, Jaquart J, Charlier R, Kessler M. Évaluation de l'implication des patients-ressources dans l'animation de séances d'éducation thérapeutique. Nephrol Ther 2015; 11:369. DOI: 10.1016/j.nephro.2015.07.327.

11. Pomey M-P, Flora L, Karazivan P, Dumez V, Lebel P, Vanier M$\mathrm{C}$, et al. Le « Montreal model» : enjeux du partenariat relationnel entre patients et professionnels de la santé, The Montreal model: the challenges of a partnership relationship between patients and healthcare professionals. Santé Publique 2015; S1:41-50.

12. Communier A, Sadki R, Sommerlatte E, Gaganyre R. Quels besoins éducatifs pour des personnes vivant avec le VIH depuis plus de 10 ans ? Educ Ther Patient - Ther Patient Educ 2010; 2:51-62.

13. Caniard E. La place des usagers dans le système de santé. Paris : Secrétariat d'État à la Santé; 2000, pp .6-7.

14. Fainzang S. La relation médecins-malades: information et mensonge. Paris : P.U.F; 2015.

15. Roux J, Charvolin F, Dumain A. Les « passions cognitives » ou la dimension rebelle du connaître en régime de passion. Rev Anthropol Connaiss 2009; 3(3):369-85.

16. Crozet C, d'Ivernois J-F. L'apprentissage de la perception des symptômes fins par des patients diabétiques: Compétence utile pour la gestion de leur maladie. Rech Educ 2010: 197-219.

17. Las Vergnas O. La culture scientifique et les non scientifiques, entre allégeance et transgression de la catégorisation scolaire [Thèse] Université de Nanterre - Paris X; 2011.

18. Epstein S. Impure Science - Aids, Activism, \& The Politics of Knowledge. Reprint. Berkeley, Calif.: University of California Press; 1998.

19. Akrich M, Méadel C, Rabeharisoa V. Se mobiliser pour la santé : Des associations de patients témoignent. 2015.

20. Harry I, Gagnayre R. Temporalité et usage des forums asynchrones dans le diabète de type 1. Contribution à l'Éducation thérapeutique du patient, Temporality and use of discussion forums in type 1 diabetes: Contribution to therapeutic patient education. Santé Publique 2013; 25:399-409.

21. Hamon T, Gagnayre R. Improving knowledge of patient skills thanks to automatic analysis of online discussions. Patient Educ Couns 2013; 92:197-204. DOI: 10.1016/j.pec.2013.05.012.

22. Brun N, Hirsch E, Kivits J, Emery G, Garcia-Viana A, Lopez A. Rapport de la mission : «Nouvelles attentes du citoyen, acteur de santé »; 2011.

23. Glasby J, Beresford P. Commentary and Issues: Who knows best? Evidence-based practice and the service user contribution. Crit Soc Policy 2006; 26:268-284. DOI: 10.1177/ 0261018306059775.

24. Weber M. Économie et société, tome 1: Les Catégories de la sociologie. Paris : Pocket; 2003.

25. Dodier N, Barbot J. Itinéraires de réparation et formation d'un espace de victimes autour d'un drame médical. Toulouse : ERES; 2011.

26. Hamel J. De la nature réflexive de la sociologie et de la disparition de son objet. Rev Eur Sci Soc 2007:45-56. DOI: 10.4000/ress. 185.

27. Campenhoudt LV, Quivy R. Manuel de recherche en sciences sociales $-4^{\mathrm{e}}$ édition. $4^{\mathrm{e}}$ édition revue et augmentée. Paris : Dunod; 2011.

28. Maturana HR. Autopoiesis and Cognition: The Realization of the Living. Softcover reprint of the original 1st ed. 1980. Dordrecht, Holland Boston: Kluwer Academic Publishers; 1979.
29. Antonovsky A. Unraveling the mystery of health: how people manage stress and stay well. 1st ed. San Francisco: Jossey-Bass; 1987.

30. Antonovsky H, Sagy S. The development of a sense of coherence and its impact on responses to stress situations. J Soc Psychol 1986; 126:213-225.

31. Gross O. L'engagement des patients au service du système de santé. Rueil-Malmaison : Doin; 2017.

32. Sen A. Development as Freedom. Oxford: Oxford University Press 1999.

33. Guibet Lafaye C. Le droit à la santé : des objectifs de l'OMS à deux ans du Millenium. Arbor 2010; 186:859-869. DOI: 10.3989/arbor.2010.745n1236.

34. Mackenzie C. Agency: un mot, un engagement. Rives Méditerranéennes 2012 35-37.

35. Sen A. Commodities and Capabilities. Amsterdam: NorthHolland; 1985.

36. Panet S, Duray-Soundron C. «C'est comme ça que ça germe ». Changement social au Sénégal : le cas de l'ONG Tostan. In: Repenser Action Collect. Une Approche Par Capab, 2008 (J-L Dubois, Ed), Paris, [Nogent-sur-Marne]: Harmattan; Réseau IMPACT.

37. Sen A. Capability and Well-Being. In: World Institute for Development Economics Research, 1993 (MC Nussbaum, A Sen, Eds), Qual. Life, Oxford [England]: New York, Clarendon Press, Oxford University Press.

38. El Harizi K. Measuring Communities Capabilities in Natural Resource Management: Methodological Notes and Findings from Marocco and Sudan. In: Repenser Action Collect. Une Approche Par Capab., 2008 (J-L Dubois, P Bakhshi, A-S Brouillet, C Duray-Soundron, Eds), Paris, Nogent-sur-Marne, Éditions L'Harmattan.

39. Ibrahim SS. From Individual to Collective Capabilities: The Capability Approach as a Conceptual Framework for Self-help. J Hum Dev 2006; 7:397-416. DOI: 10.1080/ 14649880600815982.

40. Ibrahim S. Collective Agency: Wider Freedoms and New Capabilities through Self-help. In: Repenser Action Collect. Une Approche Par Capab. (J-L Dubois, P Bakhshi, A-S Brouillet, C Duray-Soundron, editors), Paris, Nogent-sur-Marne, Éditions L'Harmattan.

41. Evans P. Collective capabilities, culture, and Amartya Sen'sDevelopment as Freedom. Stud Comp Int Dev 2002; 37:54-60. DOI: $10.1007 / B F 02686261$.

42. Nussbaum MC. Women and Human Development: The Capabilities Approach. 1st Edition. Cambridge: Cambridge University Press 2001.

43. Rabinow P. Essays on the anthropology of reason. Princeton, N.J: Princeton University Press; 1996.

44. Regulation (EC) No. 141/2000 of the European Parliament and of the Council of 16 December 1999 on orphan medicinal products.

45. Freire AMA. Pedagogia da indignação: cartas pedagógicas e outros escritos. Interface - Comun Saúde Educ 2001; 5:147-152. DOI: $10.1590 /$ S1414-32832001000100016.

46. Haraway DJ. Primate visions: gender, race, and nature in the world of modern science. New York: Routledge; 2006.

47. Puig de la Bellacasa M. Politiques féministes et construction des savoirs : «penser nous devons »! Paris : L'Harmattan 2012.

48. Rao V, Walton M. Culture and Public Action. Stanford University Press; 2004.

49. Weick KE. Sensemaking in organizations. Thousand Oaks: Sage Publications; 1995. 
50. Appadurai A. The Capacity to Aspire: Culture and the Terms of Recognition. In: Cult. Public Action. 1 edition, 2004 (V Rao, M Walton, Eds), Stanford, Calif, Stanford Social Sciences.

51. Renault E. L'expérience de l'injustice. Paris : La Découverte; 2004.

52. Mittelmark M, Sagy S, Eriksson M, Bauer G, Pelikan J, Espnes $\mathrm{G}$, editors. The handbook of salutogenesis. New York, NY: Springer Science+Business Media; 2016.

53. Sen AK. Éthique et économie et autres essais. Paris: Presses Universitaires de France; 2012.

54. Taylor C, Appiah KA, Habermas J, Rockefeller SC, Walzer M, Wolf S. Multiculturalism: Examining the Politics of Recognition. Expanded Paperback edition. Princeton, N.J: Princeton University Press; 1994.

55. Honneth A. La lutte pour la reconnaissance. Paris : Éd. du Cerf; 2002.

56. Ghadi V, Naiditch M. Comment construire la légitimité de la participation des usagers à des problématiques de santé ? Santé Publique 2006; 18:171. DOI: 10.3917/spub.062.0171.

57. Bousquet F, Ghadi V. La participation des usagers aux systèmes de santé : un tour d'horizon international. Rev Fr Aff Soc 2017: $116-127$.

58. Bates RH. Contra Contractarianism: Some Reflections on the New Institutionalism. Polit Soc 1988; 16:387-401. DOI: 10.1177/003232928801600207.

59. Gagnayre R. Pour un développement durable de l'éducation thérapeutique du patient en France. In: Traité de Santé Publique, $3^{\mathrm{e}}$ éd., 2016 (F Bourdillon, G Brücker, D Tabuteau, Eds), Paris, Lavoisier, 63-70.

60. Ricoeur P. Soi-même comme un autre. Paris : Points; 2015.

61. Prost M, Fernagu-Oudet S. L'apprenance au prisme de l'approche par les capabilités. Educ Perm 2016.
62. Compétences et socioconstructivisme: un cadre théorique. Bruxelles : De Boeck; 2012.

63. d'Ivernois J-F, Gagnayre R. Mettre en oeuvre l'éducation thérapeutique. AdSP 2001; 36:11-13.

64. Wittorski R. De la fabrication des compétences. Educ Perm 1998; 135:57-69.

65. Lallau B. Du capital social à l'agencéité : Essai sur les capacités à l'action collective des agriculteurs urbains de Kinshasha. In: Repenser l'action collective : Une approche par les capabilités, 2008 (J-L Dubois, P Bakhshi, A-S Brouillet, C DuraySoundron, C Dumbi, Eds), Paris, Nogent-sur- Marne, Éditions L'Harmattan.

66. Sen A. Rationality and freedom. 1. Paperback ed. Cambridge, Mass: Belknap Press of Harvard Univ. Press; 2004.

67. Eyerman R, Jamison A. Social movements: a cognitive approach. University Park, Pa.: Pennsylvania state university press; 1991.

68. Dietscher C, Winter U, Pelikan JM. The Application of Salutogenesis in Hospitals. In: Handb. Salut., 2017 (MB Mittelmark, S Sagy, M Eriksson, GF Bauer, JM Pelikan, B Lindström, et al., Eds), Springer International Publishing, 27798. DOI: 10.1007/978-3-319-04600-6 27.

69. Reach G. Pour l'avènement à l'hôpital d'une bientraitance ordinaire. Rev Prat 2017; 67:371-379.

70. Alaya LBFB. Le concept de «création collective de sens ». Rev Sci Gest 2011: 99-109.

71. d'Ivernois J-F, Gagnayre R. Compétences d'adaptation à la maladie du patient : une proposition. Educ Ther Patient - Ther Patient Educ 2011; 3:S201-S205. DOI: 10.1051/tpe/2011103.

72. Croyère N, Delassus E. Apprendre «du» malade. Se former à l'éducation thérapeutique. Éthique Santé 2009; 6:80-5. DOI: 10.1016/j.etiqe.2009.03.003.

Citation de l'article : Gross O, Gagnayre R. Une éducation salutogénique basée sur les capabilités des patients : une nouvelle composante de l'éducation thérapeutique. Educ Ther Patient/Ther Patient Educ 2018; 10:10501. 